OPEN ACCESS

Edited by:

Silvia Turroni,

University of Bologna, Italy

Reviewed by:

Glenn Gibson,

University of Reading, United Kingdom

Leila Khalili,

Tabriz University of Medical

Sciences, Iran

${ }^{*}$ Correspondence:

Wenyi Zhang

zhangwenyizi@163.com

†These authors have contributed equally to this work

Specialty section: This article was submitted to

Nutrition and Microbes,

a section of the journa

Frontiers in Nutrition

Received: 19 February 2021 Accepted: 17 May 2021

Published: 10 June 2021

Citation:

Peng J, Zhang M, Yao G, Kwok L-Y and Zhang $W$ (2021) Probiotics as Adjunctive Treatment for Patients

Contracted COVID-19: Current Understanding and Future Needs.

Front. Nutr. 8:669808.

doi: 10.3389/fnut.2021.669808

\section{Probiotics as Adjunctive Treatment for Patients Contracted COVID-19: Current Understanding and Future Needs}

\author{
Jiangying Peng ${ }^{1,2 t}$, Meng Zhang ${ }^{1,2 t}$, Guoqiang Yao ${ }^{1,2 t}$, Lai-Yu Kwok ${ }^{1,2}$ and Wenyi Zhang ${ }^{1,2 *}$ \\ ${ }^{1}$ Key Laboratory of Dairy Biotechnology and Engineering, Ministry of Education, Inner Mongolia Agricultural University, \\ Hohhot, China, ${ }^{2}$ Key Laboratory of Dairy Products Processing, Ministry of Agriculture and Rural Affairs, Inner Mongolia \\ Agricultural University, Hohhot, China
}

The coronavirus disease 2019 (COVID-19) is caused by a novel coronavirus, severe acute respiratory syndrome coronavirus 2 (SARS-CoV-2), which rages all over the world and seriously threatens human life and health. Currently, there is no optimal treatment for COVID-19, and emerging evidence found that COVID-19 infection results in gut microbiota dysbiosis. The intestinal microbial richness of patients of COVID-19 does not return to normal levels even six months after recovery, but probiotic adjunctive treatment has been found to restore gut homeostasis. An updated PubMed search returned four finished clinical trials that supported the use of probiotics as adjunctive treatment for COVID-19, while at least six clinical trials aiming to investigate beneficial effects of probiotic intake in managing COVID-19 are currently in progress worldwide. Here in we tentatively summarized the understanding of the actions and potential mechanisms of probiotics in the management of COVID-19. We also highlighted some future needs for probiotic researchers in the field. The success in using probiotics as adjunctive treatment for COVID-19 has expanded the scope of application of probiotics, meanwhile deepening our knowledge in the physiological function of probiotics in modulating the gut-lung axis.

Keywords: COVID-19, SARS-CoV-2, acute respiratory disease, pneumonia, probiotics, adjunctive treatment, gut-lung axis

\section{INTRODUCTION}

The coronavirus disease 2019 (COVID-19) caused by a novel coronavirus, severe acute respiratory syndrome coronavirus 2 (SARS-CoV-2), has been raged across the globe since the end of 2019, seriously threatening human life and health $(1,2)$. Yet, the number of COVID-19 cases is still growing around the world. The severity of COVID-19 has brought and will certainly continue to bring tremendous financial and medical burdens to the society; thus, cost-effective health management strategies are urgently needed.

The symptoms of COVID-19 include fever, dyspnea, dry cough, upper and/or lower respiratory tract symptoms, myalgia, fatigue, diarrhea, or a combination of these 3-6 days after exposure (3-5). Other symptoms include headache, sore throat, rhinorrhea, and gastrointestinal disorders $(4,6)$. At the beginning of the outbreak, several drugs have been applied to manage the disease, including remdesivir and favipiravir. Among them, remdesivir was found to be the most promising drug that effectively cured the first COVID-19 patient in the United States (7). Currently, multiple effective 
vaccines have been developed and are available to people worldwide. However, the decrease in gut microbiota richness and dysbiosis in patients with COVID-19 did not return to normal levels even six months after recovery, and the persistent gut dysbiosis was linked with the severity of disease and inflammatory reaction (8). Previous studies have shown that microbial diversity is a key determinant of microbial ecosystem stability $(9,10)$. A stable intestinal ecosystem enhances colonization resistance against opportunistic pathogens, while malnutrition hinders the recovery of intestinal health and lung function of patients with COVID-19 (11-13). Therefore, targeted auxiliary microecological therapy that helps restore healthy gut microbial communities is anticipated to accelerate full recovery from COVID-19 (8).

Probiotics are live microbes that, when administered in adequate amounts, confer health benefits on their hosts (14). To date, four clinical studies around the world have successfully applied probiotics as adjunctive treatment in managing COVID19 (5, 15-17), while there are more than six undergoing clinical trials (18-20). The present review summarized the understanding of the actions and potential mechanisms of probiotics in the management of COVID-19, and highlighted some future needs for probiotic researchers in the field.

\section{PROBIOTICS AS ADJUNCTIVE TREATMENT FOR PATIENTS CONTRACTED COVID-19}

In response to the outbreak of COVID-19, a Chinese infectious disease scientist proposed a treatment plan called "four-antibody, two-balance," which was successfully implemented for treating SARS-CoV-2-associated acute respiratory distress syndrome (16). The four antibodies represented antiviral, antishock, antihypertension, and anti-infection therapy, while the "twobalance" referred to acid-base balance of water electrolysis and microecological balance (16). The microecological balance was considered to be of particular importance in the treatment, as microecological imbalance would lead to secondary infection of bacteria after recovering from the viral infection (16).

An updated PubMed search recovered four finished clinical studies from different countries that found positive effects of applying probiotics as adjunctive treatment in COVID-19. For example, $\mathrm{Wu}$ et al. found that taking a large dose of probiotics as adjuvant treatment significantly improved the symptoms of the disease, accompanied by the reduction in inflammation and recovery from gut microbiota abnormalities (17). A case study report found that some COVID-19 patients suffered from microbial dysbiosis characterized by decreased levels of intestinal Lactobacillus (L.) and Bifidobacterium (B.) (16). Xu et al. observed that most COVID-19 patients with relatively mild symptoms had received probiotics (5). Baud et al. summarized data from a large body of human studies and listed different probiotics that might have relevance to lowering burden brought by COVID-19, including Lactobacillus casei, Lactobacillus plantarum, Lactobacillus rhamnosus, Lactobacillus gasseri, Bifidobacterium bifidum, Bifidobacterium longum, Bifidobacterium breve, Leuconostoc mesenteroides, and Pediococcus pentosaceus (15). Currently, there are at least six ongoing clinical trials aiming to investigate the beneficial effects of applying probiotics as adjunctive treatment in managing COVID-19 (18-20). It is noteworthy that a research scientist from Rutgers University designed a microbiota formula for early management of COVID-19 (20). This formula is a new prescription targeting to regulate the gut microbiota, aiming to prevent severe complications and hospitalization in high-risk patients of COVID-19 who suffer from underlying conditions like obesity or type-2 diabetes (20). The US Food and Drug Administration has approved the new formula for new drug clinical trial in a record time (20). Although this formula is different from ordinary probiotics-based products, the novel ecological approach focusing on managing the gut microbiota seems promising.

\section{THE UNDERSTANDING OF THE ACTIONS AND POTENTIAL MECHANISMS OF PROBIOTICS IN THE MANAGEMENT OF COVID-19}

The study of human microflora and the function of probiotics can be traced back to the longevity theory proposed by Élie Metchnikoff, a famous Russian scientist who won the Nobel Prize in 1908. Over the last decades, the advent of high-throughput sequencing technologies has revealed much information of the mechanisms behind the health-promoting effects of probiotics in maintaining gut homeostasis. A number of random clinical trials (RCTs) were performed to evaluate the beneficial role of probiotics in adjunctive treatment of various gastrointestinal diseases, such as inflammatory bowel disease, irritable bowel syndrome, and antibiotic-associated diarrhea (21). Direct and indirect evidence of health outcome data generated from various clinical trials suggested that effective probiotic adjunctive treatments rely largely on modulating the gut and lungs, and the gut-lung axis.

\section{Restoration of a Healthy Gut Microbiota}

Probiotics may enhance restoration of a stable gut microbiota in the colonic environment and prevent colonization by pathogenic organisms via a number of mechanisms $(22,23)$. For example, probiotics can bind to gut epithelial cells and result in competitive inhibition against gut wall adhesion of pathogenic microorganisms $(22,24)$. Once adhered to the gut epithelial cells, probiotics release substances that prevent the growth of pathogens and modulate intestinal permeability (22). In addition, probiotics can restore the intestinal health by attenuating inflammation and strengthening the epithelial barrier $(25,26)$. Firstly, probiotics can enhance the intestinal barrier function and prevent the invasion of pathogenic microorganisms by increasing the secretion of mucin $(25,27)$. Mucin is a highly glycosylated substance distributing in the cell membrane, and it is secreted into the intestinal cavity to form a mucus layer. The mucus layer is the first line of defense responsible for ensuring the integrity of the intestinal barrier, and pathogenic 
microorganisms would not be able to reach the gut epithelial cells without breaking through the mucus layer. Secondly, probiotics may prevent the growth of pathogens directly by secreting antibacterial factors, such as antibacterial peptides, defensins, short-chain fatty acids, and bacteriocins $(28,29)$. Thirdly, probiotics may enhance the intestinal epithelium barrier function by increasing the expression of tight junction proteins (25).

Scarce clinical data are hitherto available; however, deprivation of important gut commensals, like Lactobacillus and Bifidobacterium, has been consistently reported in some patients of COVID-19 (16). These gut microbes are known to play a significant role in maintaining colonic homeostasis. Similarly, a six-month follow-up study found that the gut microbiota richness was compromised in COVID-19 patients compared with healthy individuals (8). Higher abundances of opportunistic pathogens, including Collinsella aerofaciens, Collinsella tanakaei, Streptococcus infantis, and Morganella morganii, accompanied by an elevated functional capacity for nucleotide de novo biosynthesis, amino acid biosynthesis and glycolysis, were observed in the fecal metagenomes of high SARS-CoV-2 infectivity patients, whilst the fecal metagenomes of low-to-none SARS-CoV-2 infectivity patients were characterized by abundant shortchain fatty acid producing-bacteria, such as Parabacteroides merdae, Bacteroides stercoris, Alistipes onderdonkii, and Lachnospiraceae bacterium (30). Thus, one important goal of the probiotic adjunctive treatment in managing COVID-19 would be to restore the gut microbiota diversity, composition, and metagenomic potential to comparable levels in healthy individuals.

\section{Modulation of Host Immunity}

Probiotics are known to modulate the host immunity via regulating the innate and adapted cellular and humoral immunity $(4,31,32)$. For example, a meta-analysis of clinical trials of Lactobacillus acidopilus and L. plantarum found that these species could improve host immunity by regulating the secretion of pro-inflammatory and anti-inflammatory cytokines $(32,33)$. Thus, it is possible that the probiotic administration employed similar mechanisms to exert immunomodulatory effects to patients contracted COVID-19.

Normally, the host immunoregulation is realized by dynamic interactions between immune effector cells (e.g., dendritic cells, macrophages, and lymphocytes) and cytokines (34). Omics tools have identified probiotics-originated immunomodulatory genes and compounds (35); particularly, genes relating to the quorum-sensing system, bacteriocin biosynthesis pathways, stress responses; and compounds such as vitamins and peptides have been found to regulate cytokine production (35). It is worth noting that the clinical outcomes of probiotics-directed immunoregulatory effects are often dependent on multiple factors in the treatment protocol, including the bacterial strain, dose of application, and treatment period (36). Since only limited clinical data are available, it is yet to investigate to what extent probiotics could help maintain gut immune homeostasis in COVID-19 patients.

\section{Modulation of the Gut-Lung Axis}

Coronaviruses are divided into four genera: $\alpha, \beta, \gamma$, and $\delta$ (37). Six types of human coronaviruses were discovered before 2019, including two $\alpha$-coronaviruses, HcoV-229E and HcoV-NL63, and four $\beta$-coronaviruses, HcoV-HKU1, HcoV-OC43, severe acute respiratory syndrome coronavirus (SARS-CoV), and Middle East respiratory syndrome coronavirus (MERS-CoV). Four of them, HcoV-229E, HcoV-NL63, HcoV-HKU1, and HcoV-OC43, are usually causing mild upper respiratory tract infections (37), and they rarely invade the lower respiratory tract. In contrast, SARS$\mathrm{CoV}$ and MERS-CoV are highly homologous with SARS-CoV-2, which can spread to the lungs and cause lower respiratory tract infections and severe respiratory syndrome in humans $(38,39)$. The causative agent of the COVID-19 pandemic, SARS-CoV-2, is a $\beta$-coronavirus. It is the seventh known coronavirus that can infect humans. COVID-19 is a lower respiratory tract infection that readily spreads downward and triggers deadly levels of lung inflammation (39). The gastrointestinal tract hosts trillions of resident microorganisms, and an unstable gut microbial community can trigger an onset and/or modulate the outcome of respiratory tract infections (24). Several recent meta-analyses of prebiotic and probiotic studies have reached the same conclusion that ingesting probiotics could effectively prevent respiratory infections and reduce the number of acute respiratory infections without side effects (40-42).

Recently, the gut-lung axis concept has been established, implicating bidirectional communications and mutual dependence between these two body compartments and that alterations of the gut microbial communities may have a profound effect on lung function and disease. Experimental evidence also supports that mucosal immunity is common to different body sites. Both dendritic cells present in the gut and macrophages located in the respiratory tract can collect and present antigens to lymphocytes in nearby lymphoid tissues. Locally activated cells would then further modulate host systemic immunity by circulating through the lymphatic system. Less evidence exists showing direct transfer of microorganisms between body sites, but the transmission of intestinal bacteria to the lungs by inhaling vomit or esophageal reflux has been reported (43). In that case, the transferred microbes would be recognized by immune cells after reaching the distant body compartment, and cytokines would be released in response. Thus, the immune and inflammatory state of the gastrointestinal tract could affect other parts of the body, including the lungs (31, 43). A clinical study concluded that co-administration of oral probiotic during treatment of COVID-19 had eightfold lower risk of developing respiratory failure compared with the control subjects (41). The fact that the association of gut dysbiosis with an increased mortality in respiratory infections and severity in COVID-19-related symptoms as a result of regulatory dysfunction in inflammatory and/or antiinflammatory mechanisms in the lungs and in the gut suggests a close connection and vital cross-talks between the two mucosal compartments (44). Further longitudinal studies are needed to clarify the role of the microbiota and gut-lung cross-talks in respiratory diseases, paving the way for future application of specific probiotic strains in managing COVID-19 $(18,45,46)$. 


\section{DISCUSSION}

Changes in the gut microbiota and immunity have long been used as outcome parameters in RCTs for measuring the clinical efficacy of probiotic adjunctive treatment (47). However, the clinical outcomes described in RCTs for assessing the probiotic functions are still somewhat controversial, and the results obtained from RCTs should be cautiously interpreted because of some common limitations. For example, some RCTs were set up with a relatively small population size, hindering from obtaining a real picture of clinical efficacy and understanding of the mechanisms of the probiotic actions (22). Another plausible reason could be that the original claimed functions of the probiotics appeared to be affected by the manufacturing process (48).

Thus, in future, significantly more high-quality RCTs are necessary to achieve precise understanding of the clinical functions of probiotics as adjunctive treatments for various diseases, particularly in novel diseases like COVID-19. Proper analysis and cautious interpretation from large-scale RCTs that are soon finished will certainly help clarify the role and clinical effects of probiotics application in such cases. Moreover, stringent controls and standards should be followed in manufacturing probiotic products to ensure the product quality and genetic

\section{REFERENCES}

1. Li G, Fan Y, Lai Y, Han T, Li Z, Zhou P, et al. Coronavirus infections and immune responses. J Med Virol. (2020) 92:424-32. doi: 10.1002/jmv.25685

2. Chen N, Zhou M, Dong X, Qu J, Gong F, Han Y, et al. Epidemiological and clinical characteristics of 99 cases of 2019 novel coronavirus pneumonia in Wuhan, China: a descriptive study. Lancet. (2020) 395:507-13. doi: 10.1016/S0140-6736(20)30211-7

3. Chan JF, Yuan S, Kok KH, To KK, Chu H, Yang J, et al. A familial cluster of pneumonia associated with the 2019 novel coronavirus indicating personto-person transmission: a study of a family cluster. Lancet. (2020) 395:51423. doi: 10.1016/S0140-6736(20)30154-9

4. Sundararaman A, Ray M, Ravindra PV, Halami PM. Role of probiotics to combat viral infections with emphasis on COVID-19. Appl Microbiol Biotechnol. (2020) 104:8089-104. doi: 10.1007/s00253-02010832-4

5. Xu XW, Wu XX, Jiang XG, Xu KJ, Ying LJ, Ma CL, et al. Clinical findings in a group of patients infected with the 2019 novel coronavirus (SARSCov-2) outside of Wuhan, China: retrospective case series. BMJ. (2020) 368:m606. doi: 10.1136/bmj.m606

6. Lee IC, Huo TI, Huang YH. Gastrointestinal and liver manifestations in patients with COVID-19. J Chin Med Assoc. (2020) 83:5213. doi: 10.1097/JCMA.0000000000000319

7. Holshue ML, DeBolt C, Lindquist S, Lofy KH, Wiesman J, Bruce H, et al. First case of 2019 novel coronavirus in the United States. New Engl J Med. (2020) 382:929-36. doi: 10.1056/NEJMoa2001191

8. Chen Y, Gu S, Chen Y, Lu H, Shi D, Guo J, et al. Six-month follow-up of gut microbiota richness in patients with COVID-19. Gut. (2021) 0:13. doi: 10.1136/gutjnl-2021-324090

9. Gu S, Chen Y, Wu Z, Chen Y, Gao H, Lv L, et al. Alterations of the gut microbiota in patients with coronavirus disease 2019 or H1N1 influenza. Clin Infect Dis. (2020) 71:2669-78. doi: 10.1093/cid/ciaa709

10. Yeoh YK, Zuo T, Lui GC, Zhang F, Liu Q, Li AY, et al. Gut microbiota composition reflects disease severity and dysfunctional immune responses in patients with COVID-19. Gut. (2021) 70:698-706. doi: 10.1136/gutjnl-2020323020 stability of the cells. Last but not least, the success in using probiotics as adjunctive treatment for COVID-19 has expanded the scope of application of probiotics, which would further deepen our knowledge of the physiological function of probiotics in modulating the gut ecosystem and its interactions with the respiratory system.

\section{AUTHOR CONTRIBUTIONS}

WZ conceived the article and wrote the first draft of the article. JP, $\mathrm{MZ}$, and GY refined the structure of the article and updated the content of the article. L-YK modified the manuscript. All authors approved the final version of the manuscript.

\section{FUNDING}

This research was supported by the National Natural Science Foundation of China (Grant No. 31922071).

\section{ACKNOWLEDGMENTS}

We would like to thank the other postgraduate students in the laboratory for their assistance in retrieving the literature.
11. Lahti L, Salojärvi J, Salonen A, Scheffer M, de Vos WM. Tipping elements in the human intestinal ecosystem. Nat Commun. (2014) 5:4344. doi: 10.1038/ncomms5344

12. Dickson RP, Singer BH, Newstead MW, Falkowski NR, Erb-Downward JR, Standiford TJ, et al. Enrichment of the lung microbiome with gut bacteria in sepsis and the acute respiratory distress syndrome. Nat Microbiol. (2016) 1:16113. doi: 10.1038/nmicrobiol.2016.113

13. Zhang Q, Ran X, He Y, Ai Q, Shi Y. Acetate downregulates the activation of NLRP3 inflammasomes and attenuates lung injury in neonatal mice with bronchopulmonary dysplasia. Front Pediatr. (2020) 8:595157. doi: 10.3389/fped.2020.595157

14. Hill C, Guarner F, Reid G, Gibson GR, Merenstein DJ, Pot B, et al. Expert consensus document. The International scientific association for probiotics and prebiotics consensus statement on the scope and appropriate use of the term probiotic. Nat Rev Gastroenterol Hepatol. (2014) 11:50614. doi: 10.1038/nrgastro.2014.66

15. Baud D, Dimopoulou Agri V, Gibson GR, Reid G, Giannoni E. Using probiotics to flatten the curve of coronavirus disease COVID-2019 pandemic. Front Public Health. (2020) 8:186. doi: 10.3389/fpubh.2020.00186

16. Xu K, Cai H, Shen Y, Ni Q, Chen Y, Hu S, et al. [Management of corona virus disease-19 (COVID-19): the Zhejiang experience]. Zhejiang Da Xue Xue Bao Yi Xue Ban. (2020) 49:147-57. doi: 10.3785/j.issn.1008-9292.2020.02.02

17. Chunyan W, Qian X, Zhan C, Dengdeng P, Ying Z, Sheng W, et al. The volatile and heterogenous gut microbiota shifts of COVID-19 patients over the course of a probiotics-assisted therapy. Res Square. (2021) 0:116. doi: 10.21203/rs.3.rs-72753/v1

18. Baindara P, Chakraborty R, Holliday ZM, Mandal SM, Schrum AG. Oral probiotics in coronavirus disease 2019: connecting the gut-lung axis to viral pathogenesis, inflammation, secondary infection and clinical trials. New Microb New Infect. (2021) 40:100837. doi: 10.1016/j.nmni.2021.100837

19. Hu J, Zhang L, Lin W, Tang W, Chan FKL, Ng SC. Review article: probiotics, prebiotics and dietary approaches during COVID-19 pandemic. Trends Food Sci Technol. (2021) 108:187-96. doi: 10.1016/j.tifs.2020.12.009

20. Zhao L. Rutgers Researcher Invents Microbiota Formula to Help High Risk Patients Fight COVID-19. New Brunswick, NJ: Rutgers The State University of New Jersey (2021). 
21. Suvorov A. Gut microbiota, probiotics, human health. Biosci Microb Food Health. (2013) 32:81-91. doi: 10.12938/bmfh.32.81

22. Ding RX, Goh WR, Wu RN, Yue XQ, Luo X, Khine WWT, et al. Revisit gut microbiota and its impact on human health and disease. J Food Drug Anal. (2019) 27:623-31. doi: 10.1016/j.jfda.2018.12.012

23. Ferreira C, Viana SD, Reis F. Gut microbiota dysbiosis-immune hyperresponse-inflammation triad in coronavirus disease 2019 (COVID-19): impact of pharmacological and nutraceutical approaches. Microorganisms. (2020) 8:1514. doi: 10.3390/microorganisms8101514

24. Walton GE, Gibson GR, Hunter KA. Mechanisms linking the human gut microbiome to prophylactic and treatment strategies for COVID-19. $\mathrm{Br}$ J Nutr. (2020) 0:1-9. doi: 10.1017/S0007114520003980

25. Hiippala $K$, Jouhten $H$, Ronkainen A, Hartikainen A, Kainulainen $\mathrm{V}$, Jalanka J, et al. The potential of gut commensals in reinforcing intestinal barrier function and alleviating inflammation. Nutrients. (2018) 10:988. doi: 10.3390/nu10080988

26. Giannoni E, Baud D, Agri VD, Gibson GR, Reid G. Probiotics and COVID-19. Lancet Gastroenterol Hepatol. (2020) 5:7201. doi: 10.1016/S2468-1253(20)30195-3

27. Din AU, Mazhar M, Waseem M, Ahmad W, Bibi A, Hassan A, et al. SARS-CoV-2 microbiome dysbiosis linked disorders and possible probiotics role. Biomed Pharmacother. (2021) 133:110947. doi: 10.1016/j.biopha.2020. 110947

28. Yu LC, Wang JT, Wei SC, Ni YH. Host-microbial interactions and regulation of intestinal epithelial barrier function: from physiology to pathology. World J Gastrointest Pathophysiol. (2012) 3:27-43. doi: 10.4291/wjgp. v3.i1.27

29. Yang Q, Liang Q, Balakrishnan B, Belobrajdic DP, Feng QJ, Zhang W. Role of dietary nutrients in the modulation of gut microbiota: a narrative review. Nutrients. (2020) 12:381. doi: 10.3390/ nu12020381

30. Zuo T, Liu Q, Zhang F, Lui GC, Tso EY, Yeoh YK, et al. Depicting SARSCoV-2 faecal viral activity in association with gut microbiota composition in patients with COVID-19. Gut. (2021) 70:276-84. doi: 10.1136/gutjnl-2020322294

31. Stavropoulou E, Bezirtzoglou E. Probiotics as a weapon in the fight against COVID-19. Front Nutr. (2020) 7:614986. doi: 10.3389/fnut.2020.614986

32. Zhao W, Peng C, Sakandar HA, Kwok LY, Zhang W. Metaanalysis: randomized trials of Lactobacillus plantarum on immune regulation over the last decades. Front Immunol. (2021) 12:643420. doi: 10.3389/fimmu.2021.643420

33. Zhao W, Liu Y, Kwok L-Y, Cai T, Zhang W. The immune regulatory role of Lactobacillus acidophilus: an updated meta-analysis of randomized controlled trials. Food Biosci. (2020) 36:100656. doi: 10.1016/j.fbio.2020. 100656

34. Ya T, Zhang Q, Chu F, Merritt J, Bilige M, Sun T, et al. Immunological evaluation of Lactobacillus casei Zhang: a newly isolated strain from koumiss in inner Mongolia, China. BMC Immunol. (2008) 9:68. doi: 10.1186/1471-2172-9-68

35. Yan F, Polk DB. Probiotics and immune health. Curr Opin Gastroenterol. (2011) 27:496-501. doi: 10.1097/MOG.0b013e32834baa4d

36. Kang HJ, Im SH. Probiotics as an immune modulator. J Nutr Sci Vitaminol. (2015) 61:S103-5. doi: 10.3177/jnsv.61.S103
37. Chan JF, Lau SK, To KK, Cheng VC, Woo PC, Yuen KY. Middle East respiratory syndrome coronavirus: another zoonotic betacoronavirus causing SARS-like disease. Clin Microbiol Rev. (2015) 28:465-522. doi: 10.1128/CMR.00102-14

38. Gaunt ER, Hardie A, Claas EC, Simmonds P, Templeton KE. Epidemiology and clinical presentations of the four human coronaviruses 229E, HKU1, NL63, and OC43 detected over 3 years using a novel multiplex real-time PCR method. J Clin Microbiol. (2010) 48:2940-7. doi: 10.1128/JCM.00636-10

39. Duarte R, Furtado I, Sousa L, Carvalho CFA. The 2019 novel coronavirus (2019-nCoV): novel virus, old challenges. Acta Med Port. (2020) 33:155. doi: 10.20344/amp.13547

40. Wang Y, Li X, Ge T, Xiao Y, Liao Y, Cui Y, et al. Probiotics for prevention and treatment of respiratory tract infections in children: a systematic review and meta-analysis of randomized controlled trials. Medicine. (2016) 95:e4509. doi: 10.1097/MD.0000000000004509

41. d'Ettorre G, Ceccarelli G, Marazzato M, Campagna G, Pinacchio C, Alessandri F, et al. Challenges in the management of SARS-CoV2 infection: the role of oral bacteriotherapy as complementary therapeutic strategy to avoid the progression of COVID-19. Front Med. (2020) 7:389. doi: 10.3389/fmed.2020.00389

42. Hao Q, Dong BR, Wu T. Probiotics for preventing acute upper respiratory tract infections. Cochr Database Syst Rev. (2015) 2:CD006895. doi: 10.1002/14651858.CD006895.pub3

43. Marsland BJ, Trompette A, Gollwitzer ES. The gut-lung axis in respiratory disease. Ann Am Thor Soc. (2015) 12(Suppl. 2):S150-6. doi: 10.1513/AnnalsATS.201503-133AW

44. de Oliveira GLV, Oliveira CNS, Pinzan CF, de Salis LVV, Cardoso CRB. Microbiota modulation of the gut-lung axis in COVID-19. Front Immunol. (2021) 12:635471. doi: 10.3389/fimmu.2021.635471

45. Akour A. Probiotics and COVID-19: is there any link? Lett Appl Microbiol. (2020) 71:229-34. doi: 10.1111/lam.13334

46. Mak JWY, Chan FKL, Ng SC. Probiotics and COVID-19: one size does not fit all. Lancet Gastroenterol Hepatol. (2020) 5:644-45. doi: 10.1016/S2468-1253(20)30122-9

47. Dickerson F, Adamos M, Katsafanas E, Khushalani S, Origoni A, Savage C, et al. Adjunctive probiotic microorganisms to prevent rehospitalization in patients with acute mania: a randomized controlled trial. Bipolar Disord. (2018) 20:614-21. doi: 10.1111/bdi.12652

48. Sybesma W, Molenaar D, van IJcken W, Venema K, Kort R. Genome instability in Lactobacillus rhamnosus GG. Appl Environ Microbiol. (2013) 79:2233-9. doi: 10.1128/AEM.03566-12

Conflict of Interest: The authors declare that the research was conducted in the absence of any commercial or financial relationships that could be construed as a potential conflict of interest.

Copyright (C) 2021 Peng, Zhang, Yao, Kwok and Zhang. This is an open-access article distributed under the terms of the Creative Commons Attribution License (CC BY). The use, distribution or reproduction in other forums is permitted, provided the original author(s) and the copyright owner(s) are credited and that the original publication in this journal is cited, in accordance with accepted academic practice. No use, distribution or reproduction is permitted which does not comply with these terms. 